\title{
Echocardiographic parameters and renal outcomes in patients with preserved renal function, and mild- moderate CKD
}

\author{
Thomas A. Mavrakanas ${ }^{1,2^{*}} \mathbb{D}$, Aisha Khattak ${ }^{1,3 \dagger}$, Karandeep Singh ${ }^{4}$ and David M. Charytan ${ }^{1}$
}

\begin{abstract}
Background: Echocardiographic characteristics across the spectrum of chronic kidney disease (CKD) have not been well described. We assessed the echocardiographic characteristics of patients with preserved renal function and mild or moderate CKD referred for echocardiography and determined whether echocardiographic parameters of left ventricular (LV) and right ventricular (RV) structure and function were associated with changes in renal function and mortality.

Methods: This retrospective cohort study enrolled all adult patients who had at least one trans-thoracic echocardiography between 2004 and 2014 in our institution. The composite outcome of doubling of serum creatinine or initiation of maintenance dialysis or kidney transplantation was the primary outcome. Mortality was the secondary outcome.

Results: 29,219 patients were included. Patients with worse renal function had higher prevalence of structural and functional LV and RV abnormalities. Higher estimated glomerular filtration rate (eGFR) was independently associated with preserved LV ejection fraction, preserved RV systolic function, and lower LV mass, left atrial diameter, pulmonary artery pressure, and right atrial pressure, as well as normal RV structure. 1041 composite renal events were observed. 8780 patients died during the follow-up. Pulmonary artery pressure and the RV, but not the LV, echocardiographic parameters were independently associated with the composite renal outcome. In contrast, RV systolic function, RV dilation or hypertrophy, LV ejection fraction group, LV diameter quartile, and pulmonary artery pressure quartile were independently associated with all-cause mortality.
\end{abstract}

Conclusions: Echocardiographic abnormalities are frequent even in early CKD. Echocardiographic assessment particularly of the RV may provide useful information for the care of patients with CKD.

Keywords: Echocardiography, Chronic kidney disease, Doubling of serum creatinine, Dialysis, Kidney transplantation, Mortality

\section{Background}

Chronic kidney disease (CKD) is highly prevalent among patients with congestive heart failure (CHF) and is associated with higher mortality rates [1]. In the CHARM trials, for example, an estimated glomerular filtration rate $($ eGFR $)<60 \mathrm{ml} / \mathrm{min} / 1.73 \mathrm{~m}^{2}$ was an independent

\footnotetext{
* Correspondence: tmavrakanas@partners.org; tmavrakanas@gmail.com tThomas A. Mavrakanas and Aisha Khattak contributed equally to this work. ${ }^{1}$ Renal Division, Brigham \& Women's Hospital, Harvard Medical School, Boston, MA, USA

${ }^{2}$ Division of Nephrology, Department of Medicine, McGill University, Montreal, Canada

Full list of author information is available at the end of the article
}

predictor of cardiovascular death or admission for decompensated heart failure [2].

Echocardiography can identify abnormalities in heart function or structure that are associated with kidney dysfunction [3]. Abnormal left ventricular (LV) geometry, lower mid-wall fractional shortening, and higher LV mass index have been described among patients with CKD compared with patients with preserved renal function in a cohort of participants with heart failure with preserved ejection fraction [3]. In other studies, echocardiographic parameters predicted cardiovascular events among CKD patients, although there was no net reclassification

(C) The Author(s). 2018 Open Access This article is distributed under the terms of the Creative Commons Attribution 4.0 International License (http://creativecommons.org/licenses/by/4.0/), which permits unrestricted use, distribution, and 
improvement when these parameters were added to a model including clinical and laboratory variables [4].

Although these data are intriguing, the echocardiographic characteristics of patients across the spectrum of cardiac function - with both preserved or reduced LV ejection fraction and with or without CHF - have not been adequately described across different eGFR stages, especially in patients with mildly decreased eGFR. It is also uncertain whether echocardiographic parameters predict CKD progression. The objective of this study was to describe the echocardiographic characteristics of patients with preserved renal function and mild or moderate CKD referred for echocardiography, and to assess associations of echocardiographic parameters with changes in renal function.

\section{Methods}

\section{Study population}

Patients were included if they had at least one trans-thoracic echocardiography with tissue Doppler imaging at a Partners affiliated healthcare facility between January 1st 2004 and January 6th 2014, were $\geq 18$ years old at the time of echocardiography, and had at least one eGFR between 30 and $120 \mathrm{ml} / \mathrm{min} / 1.73 \mathrm{~m}^{2}$ during this time period. For patients who had more than one echocardiogram, the first one was used.

Patients on maintenance dialysis or a history of kidney or heart transplant were excluded, as were those without creatinine values from 365 days before to 90 days after echocardiography.

The study was designed to capture patients with preserved renal function and mild or moderate CKD $\left(\mathrm{eGFR} \geq 90,60-89,30-59 \mathrm{ml} / \mathrm{min} / 1.73 \mathrm{~m}^{2}\right)$.

\section{Data collection}

The Research Patient Data registry (RPDR), a registry of all patients followed at a Partners healthcare facility, was used to retrieve all encounters, medication prescriptions, laboratory tests, and echocardiography results conducted during this time period.

To avoid misdiagnosis of acute kidney injury at the time of baseline echocardiography as CKD, the average baseline creatinine was calculated using all the available creatinines for each patient from 365 days prior to 90 days after the index echocardiogram (16 values on average, median of 7). Baseline eGFR was calculated using the CKD-EPI equation.

\section{Comorbidities and baseline data}

Information on comorbidities was extracted using condition-specific diagnostic codes (International Classification of Diseases, 9th revision-Additional file 1 Table S1). Comorbidities were diagnosed on the basis of relevant inpatient or outpatient diagnoses preceding the echocardiography date. As many echocardiograms are performed during a first admission for decompensated heart failure, any patient with a first CHF diagnostic code in the 30 days after the echocardiogram was considered to have baseline CHF.

The following baseline laboratory data were extracted (the values closest to the echocardiogram date, from 365 days before to 90 days after echocardiography): hemoglobin, albumin, calcium, phosphorus, urine albumin to creatinine ratios, and brain natriuretic peptide (BNP) levels.

We determined the use of angiotensin converting enzyme inhibitors (ACEI), angiotensin receptor blockers (ARB), $\beta$-blockers, statins, aspirin, clopidogrel, and warfarin by matching the most recent outpatient medication lists with generic and brand name drugs.

\section{Echocardiograms}

The following parameters were extracted from the echocardiography reports using automated text processing: LV ejection fraction, LV diastolic diameter, LV posterior wall thickness, interventricular septal thickness in diastole, LV mass (area-length method), LV mass index (corrected for body surface area), pulmonary artery pressure, right atrial pressure, left atrium diameter, aortic root diameter, concentric hypertrophy and regional wall motion abnormalities, right ventricular (RV) systolic function (preserved or reduced), and RV hypertrophy or dilatation (presence or absence). All echocardiogram reports were acquired and recorded using an American Society of Echocardiography-recommended scanning protocol with standard techniques. Although we initially planned to examine associations of diastolic dysfunction with CKD progression, tissue Doppler imaging results were image-embedded and not readily extractable.

We randomly reviewed 100 patient records to check the accuracy of automated extraction of diagnoses, medication, and echocardiographic parameters. Specificity was $100 \%$ and sensitivity was between 95 and $97 \%$ for the different echocardiographic parameters (most available information was captured using regular expression searches).

\section{Outcomes}

We retrospectively searched in the registry for available creatinine values or diagnostic codes post index echocardiography. The composite of doubling of serum creatinine, maintenance dialysis or kidney transplantation was the primary outcome because it is a standard, FDA-validated outcome. Mortality was the secondary outcome. We also analyzed associations between echocardiographic parameters and eGFR, using the latter as the exposure.

Clinical outcomes were identified from RPDR using diagnostic codes. The RPDR is continuously matched to the social security death index, a national database 
including all deaths in the United States. For the outcome of doubling of serum creatinine, creatinine values starting 30 days after the first doubling as well as the last available creatinine were screened for repeated/confirmed doubling to rule out potential AKI. Patients were censored at the last clinical visit or lab test.

\section{Statistical analysis}

Baseline characteristics across eGFR groups were compared using the $x^{2}$ test for categorical variables, and one-way analysis of variance or non-parametric trend tests for continuous variables.

In order to study the association between echocardiographic parameters and eGFR, we used linear regression for continuous echocardiographic variables or logistic regression for binary variables. Normality, linearity, and homoscedasticity assumptions were confirmed using graphical methods. The following covariates were included in multivariate models on the basis of known associations with the outcomes of interest, clinical intuition, and availability in the dataset: age, sex, race, history of hypertension, diabetes, coronary disease, CHF, and use of ACEI or ARB.

In order to assess event-free survival, the cohort was divided into quartiles for LV diastolic diameter, LV mass, LV mass index, left atrium diameter, and pulmonary artery pressure. Categorical variables or variables reported in intervals rather than as true continuous measurements were assessed in binary categories (RV systolic function, RV hypertrophy and dilation, and right atrial pressure) except for LV ejection fraction where the following cutoffs were used: <25\%, 25-39\%, 40-54\%, $\geq 55 \%$. The incidence of the composite renal outcome was calculated for each quartile. The $x^{2}$ test was used to compare the different groups. To assess associations of echocardiographic parameters with clinical outcomes, multivariable Cox proportional hazards regression was used. Proportional hazards assumptions were verified using graphical methods. Patients without available creatinine values or diagnostic codes for dialysis or transplantation were considered to have stable renal function. The following parameters were included in the baseline model: age, sex, race, baseline eGFR, history of hypertension, diabetes, coronary artery disease, CHF, and use of ACEI or ARB. The various echocardiographic parameters were then added to the model. Patients with missing echocardiographic parameters were excluded from the respective survival analysis.

We conducted three sensitivity analyses in the following subgroups: i) outpatients only-in order to exclude individuals with echocardiograms during an acute cardiac event; ii) individuals without CHF at baseline; iii) individuals with available follow-up creatinine values or diagnostic codes.
$P$-values $<0.05$ were considered statistically significant. SPSS software (version 20.0, SPSS Inc., Chicago, IL, USA) was used for all statistical analyses.

\section{Results}

Echocardiographic parameters in different eGFR groups

We identified 42,446 patients with at least one echocardiogram and one eGFR value between 30 and $120 \mathrm{ml} / \mathrm{min} /$ $1.73 \mathrm{~m}^{2}$. Of these, $31,173 \mathrm{had}$ at least one creatinine value available from 365 days before to 90 days after the echocardiogram. 492 patients with a transplanted heart or kidney, 268 patients on dialysis at baseline, and 1194 patients who had a baseline eGFR $<30 \mathrm{ml} / \mathrm{min} / 1.73 \mathrm{~m}^{2}$ were excluded from the main analysis. The characteristics of the 29,219 patients with an eGFR $\geq 30 \mathrm{ml} / \mathrm{min} / 1.73 \mathrm{~m}^{2}$ at baseline are depicted in Table 1 . Mean time between echocardiography and serum creatinine was $2.3 \pm 0.1$ days. Patients with worse renal function were older, more commonly white, had a higher prevalence of hypertension, diabetes, coronary artery disease, or heart failure, higher urine albumin to creatinine ratios, and higher potassium and phosphorus levels. BNP levels were also higher with increasing creatinine.

Patients with worse renal function had lower LV ejection fraction, higher prevalence of RV systolic impairment, higher LV mass index, higher LV and left atrium diameter, more regional wall motion abnormalities, and higher prevalence of RV hypertrophy or dilated RV (Table 2). They also had higher pulmonary artery pressures, and higher prevalence of elevated right atrial pressure. eGFR was independently associated with all echocardiographic parameters analyzed in multi-variable models (Additional file 2 Table S2).

\section{Echocardiographic parameters in patients with and without history of CHF}

A total of 8183 patients had history of CHF: 1733 (21\%) with an eGFR $\geq 90,3369$ (41\%) with an eGFR of $60-89$, and $3081(38 \%)$ with an eGFR $<60 \mathrm{ml} / \mathrm{min} / 1.73 \mathrm{~m}^{2}$. The baseline and echocardiographic characteristics according to eGFR groups of patients with CHF are shown in Additional file 3: Table S3 and Additional file 4: Table S4.

In the subgroup of patients without CHF, prevalence of various comorbidities at baseline was lower compared with patients with CHF. In those without CHF, right atrial pressure levels were similar in all three eGFR groups. For all the other echocardiographic parameters, a significant linear trend was identified across the different eGFR groups but the prevalence of RV or LV systolic dysfunction was lower (Additional file 5: Table S5 and Additional file 6: Table S6).

\section{Renal outcomes}

At least one follow-up creatinine (beyond day 90 from the index echocardiogram) or diagnostic code for 
Table 1 Baseline characteristics of the study patients

\begin{tabular}{|c|c|c|c|c|c|c|}
\hline \multirow[t]{2}{*}{ Characteristic } & \multicolumn{2}{|c|}{ eGFR 90-120 } & \multicolumn{2}{|c|}{ eGFR 60-89 } & \multicolumn{2}{|c|}{ eGFR 30-59 } \\
\hline & $\mathrm{N}$ & Result & $\mathrm{N}$ & Result & $\mathrm{N}$ & Result \\
\hline Patients & & 9864 (34\%) & & $12,731(43 \%)$ & & $6624(23 \%)$ \\
\hline Outpatients & & 4558 (46\%) & & $6124(48 \%)$ & & 2448 (37\%) \\
\hline Age (years) & 9864 & $50 \pm 14$ & 12,731 & $64 \pm 13$ & 6624 & $72 \pm 12$ \\
\hline Male sex & 9864 & 4797 (49\%) & 12,731 & 6608 (52\%) & 6624 & 3281 (50\%) \\
\hline African American & 9864 & 1168 (12\%) & 12,731 & $949(8 \%)$ & 6624 & $523(8 \%)$ \\
\hline Hypertension & 9864 & 3480 (35\%) & 12,731 & $6884(54 \%)$ & 6624 & 4091 (62\%) \\
\hline Diabetes mellitus & 9864 & 1308 (13\%) & 12,731 & 2307 (18\%) & 6624 & 1902 (29\%) \\
\hline$C A D$ & 9864 & 3087 (31\%) & 12,731 & $5984(47 \%)$ & 6624 & 3762 (57\%) \\
\hline $\mathrm{CHF}$ & 9864 & $1733(18 \%)$ & 12,731 & 3369 (27\%) & 6624 & 3081 (47\%) \\
\hline COPD & 9864 & $286(3 \%)$ & 12,731 & $454(4 \%)$ & 6624 & $319(5 \%)$ \\
\hline PE & 9864 & $520(5 \%)$ & 12,731 & 452 (4\%) & 6624 & $257(4 \%)$ \\
\hline ACEI & 8953 & $1614(18 \%)$ & 11,642 & 3278 (28\%) & 6175 & 2222 (36\%) \\
\hline ARB & 8953 & $330(4 \%)$ & 11,642 & $928(8 \%)$ & 6175 & 810 (13\%) \\
\hline$\beta$-blocker & 8953 & $3618(40 \%)$ & 11,642 & 6190 (53\%) & 6175 & 3902 (63\%) \\
\hline Statin & 8953 & $2298(26 \%)$ & 11,642 & 5077 (44\%) & 6175 & 3317 (54\%) \\
\hline Aspirin & 8953 & 2993 (33\%) & 11,642 & 5287 (45\%) & 6175 & 3373 (55\%) \\
\hline Clopidogrel & 8953 & 659 (7\%) & 11,642 & 1325 (11\%) & 6175 & $844(14 \%)$ \\
\hline Warfarin & 8953 & $940(11 \%)$ & 11,642 & $1852(16 \%)$ & 6175 & 1168 (19\%) \\
\hline Creatinine $(\mu \mathrm{mol} / \mathrm{l})$ & 9864 & $65(57-74)$ & 12,731 & $82(73-94)$ & 6624 & 115 (100-134) \\
\hline eGFR $\left(\mathrm{ml} / \mathrm{min} / 1.73 \mathrm{~m}^{2}\right)$ & 9864 & $104 \pm 12$ & 12,731 & $76 \pm 9$ & 6624 & $47 \pm 8$ \\
\hline \multicolumn{7}{|l|}{ Albuminuria stage: } \\
\hline$-A 1$ & 348 & $213(61 \%)$ & 544 & $333(61 \%)$ & 472 & $188(40 \%)$ \\
\hline$-A 2$ & 348 & $100(29 \%)$ & 544 & $159(29 \%)$ & 472 & 191 (41\%) \\
\hline$-A 3$ & 348 & $35(10 \%)$ & 544 & $52(10 \%)$ & 472 & $93(20 \%)$ \\
\hline $\mathrm{Hb}(\mathrm{g} / \mathrm{l})$ & 7115 & $123 \pm 23$ & 8458 & $127 \pm 21$ & 4301 & $118 \pm 21$ \\
\hline Albumin (g/l) & 7331 & $38 \pm 7$ & 9261 & $39 \pm 6$ & 5183 & $37 \pm 6$ \\
\hline $\mathrm{K}^{+}(\mathrm{mmol} / \mathrm{l})$ & 9522 & $4.0 \pm 0.4$ & 12,312 & $4.0 \pm 0.4$ & 6448 & $4.1 \pm 0.5$ \\
\hline Calcium (mmol/l) & 8491 & $2.20 \pm 0.18$ & 10,961 & $2.23 \pm 0.15$ & 5796 & $2.23 \pm 0.18$ \\
\hline Phos (mmol/l) & 3974 & $1.0(0.8-1.2)$ & 4188 & $1.0(0.9-1.2)$ & 2917 & $1.1(0.9-1.2)$ \\
\hline BNP (ng/L) & 906 & $90(27-265)$ & 1610 & $188(62-480)$ & 1519 & 349 (137-745) \\
\hline
\end{tabular}

Results are presented as number (percentage), mean \pm standard deviation, or median (interquartile range). eGFR, estimated glomerular filtration rate; $\mathrm{N}$, number of patients with available data; $C A D$, coronary artery disease; $C H F$, congestive heart failure; COPD, chronic obstructive lung disease; $P E$, pulmonary embolism (acute or chronic); ACEl, angiotensin converting enzyme inhibitor; ARB, angiotensin receptor blocker; A1: albuminuria < $30 \mathrm{mg} / \mathrm{g}$ of creatinine; A2: albuminuria 30-300 mg/g of creatinine; A3: albuminuria $>300 \mathrm{mg} / \mathrm{g}$ of creatinine; $\mathrm{Hb}$, hemoglobin; $\mathrm{K}^{+}$, potassium; Phos, phosphorus; BNP, brain natriuretic peptide. Percentages are within eGFR group and exclude missing values. The three eGFR groups were statistically different ( $\mathrm{P}$ value for trend $<0.001$ for all parameters except sex)

dialysis/transplantation were available for 20,008 patients (68\%). Median follow up was 48 months (interquartile range 20-87 months). A total of 1041 composite renal events were observed: 691 events with the creatinine doubling, 273 initiations of dialysis, and 77 kidney transplantations; 282 occurred in patients with an eGFR $\geq 90$ (0.86 events per 100 patients-years), 348 in patients with eGFR between 60 and 89 (0.73 events per 100 patients-years), and 411 in patients with an eGFR $<60 \mathrm{ml} / \mathrm{min} / 1.73 \mathrm{~m}^{2}$ (1.79 events per 100 patients-years).
The number of composite renal events that occurred in the 1st, 2nd, 3rd, and 4th quartile of each echocardiographic parameter is depicted in Fig. 1. An increase was observed in higher quartiles of LV and left atrial diameter, LV mass and pulmonary artery pressure but not of LV mass index. Lower LV ejection fraction groups also had higher renal event rates. However, in adjusted models, most LV parameters were not independently associated with the composite renal outcome, with the exception of the second quartile of LV diastolic diameter 
Table 2 Echocardiographic characteristics of the study patients

\begin{tabular}{|c|c|c|c|c|c|c|}
\hline \multirow[b]{2}{*}{ Characteristic } & \multicolumn{2}{|c|}{ eGFR 90-120 } & \multicolumn{2}{|c|}{ eGFR 60-89 } & \multicolumn{2}{|c|}{ eGFR 30-59 } \\
\hline & $\mathrm{N}$ & Result & $\mathrm{N}$ & Result & $\mathrm{N}$ & Result \\
\hline Preserved EF & 9161 & 7604 (83\%) & 11,730 & 8975 (77\%) & 6192 & 4058 (66\%) \\
\hline LVEF (\%) & 9161 & $60(55-60)$ & 11,730 & $60(55-60)$ & 6192 & $55(45-60)$ \\
\hline $\mathrm{LVd}(\mathrm{cm})$ & 7576 & $4.66 \pm 0.72$ & 9562 & $4.64 \pm 0.80$ & 5411 & $4.70 \pm 0.93$ \\
\hline LVPW (cm) & 5247 & $0.98 \pm 0.22$ & 6141 & $1.04 \pm 0.23$ & 3431 & $1.09 \pm 0.25$ \\
\hline IVSd (cm) & 6918 & $1.02 \pm 0.24$ & 8759 & $1.10 \pm 0.25$ & 4975 & $1.15 \pm 0.26$ \\
\hline LVM (g) & 3853 & $161(126-205)$ & 4229 & $174(135-223)$ & 2377 & $186(146-243)$ \\
\hline LVMi $\left(\mathrm{g} / \mathrm{m}^{2}\right)$ & 2857 & 85 (69-103) & 3204 & $92(74-115)$ & 1705 & $102(81-128)$ \\
\hline LAd (cm) & 7299 & $3.66 \pm 0.72$ & 9277 & $3.92 \pm 0.78$ & 5245 & $4.14 \pm 0.83$ \\
\hline PAP (mmHg) & 5309 & $22(18-28)$ & 7385 & $25(19-31)$ & 4359 & $28(22-37)$ \\
\hline RAP $>6 \mathrm{~cm} \mathrm{H} \mathrm{H}_{2} \mathrm{O}$ & 3831 & $283(7 \%)$ & 4400 & 449 (10\%) & 2317 & 372 (16\%) \\
\hline Impaired RV systolic function & 7810 & $430(6 \%)$ & 9887 & $789(8 \%)$ & 5376 & $717(13 \%)$ \\
\hline Increased RV diameter & 7982 & $728(9 \%)$ & 10,148 & $1114(11 \%)$ & 5555 & $907(16 \%)$ \\
\hline RV hypertrophy & 4990 & $117(2 \%)$ & 5888 & $171(3 \%)$ & 2840 & $172(6 \%)$ \\
\hline $\mathrm{ARd}(\mathrm{cm})$ & 6946 & $3.09 \pm 0.47$ & 8927 & $3.18 \pm 0.47$ & 5052 & $3.18 \pm 0.48$ \\
\hline RWMA & 6320 & $348(6 \%)$ & 7366 & $718(10 \%)$ & 3377 & $540(16 \%)$ \\
\hline
\end{tabular}

Results are presented as number (percentage), mean \pm standard deviation, or median (interquartile range). N, number of patients with available data; LVEF, left ventricular ejection fraction; EF, ejection fraction; LVd, left ventricular diastolic diameter; LVPW, left ventricular posterior wall thickness; IVSd, interventricular septal thickness in diastole; LVM, left ventricular mass (area-length method); LVMi, left ventricular mass index (corrected for body surface area); LAd, left atrium diameter; PAP, pulmonary artery pressure; RAP, right atrial pressure; RV, right ventricle; ARd, aortic root diameter; RWMA, regional wall motion abnormalities. Percentages exclude missing values. The three eGFR groups were statistically different ( $P$ value for trend $<0.001$ for all parameters except LVd; $p=0.01$ for $L V d$ )

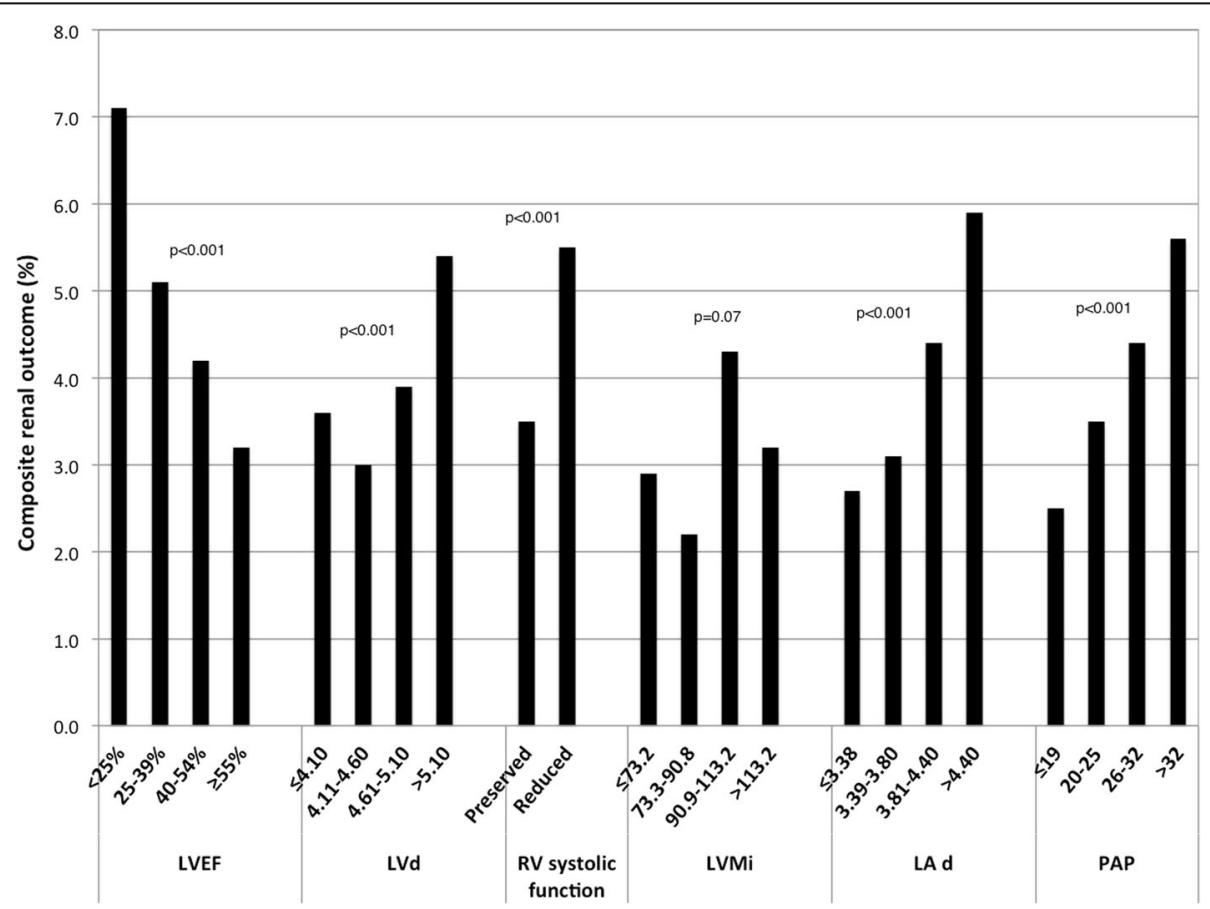

Fig. 1 Percentage of patients with CKD progression by category of echocardiographic parameters. The incidence of the composite renal outcome (doubling of serum creatinine or initiation of maintenance dialysis or kidney transplantation) was calculated for each quartile of LVd, LVM, LVMi, LAd, and PAP. For LVEF, the following cutoffs were used: <25\%, 25-39\%, 40-54\%, $255 \%$. P values are for trend. LVEF, left ventricular ejection fraction; LVd, left ventricular diastolic diameter; RV, right ventricular; LVMi, left ventricular mass index (corrected for body surface area); LA d, left atrium diameter; PAP, pulmonary artery pressure. P for trend provided 
(Table 3). In contrast, pulmonary artery pressure and RV systolic function and dilation were independent predictors of the composite renal outcome. The adjusted HR for impaired RV systolic function was 1.51 (95\% CI 1.21-1.88, $p<0.001$ ), and for RV dilation 1.59 (95\% CI 1.32-1.92, $\mathrm{p}<0.001)$.

\section{Mortality}

8780 patients died during the follow-up: 2118 (24\% of deaths) among patients with an eGFR $\geq 90$ (6.4 events per 100 patients-years), 3456 (39\% of deaths) among patients with eGFR between 60 and 89 (7.2 events per 100 patients-years), and 3206 (37\% of deaths) among patients with eGFR $<60 \mathrm{ml} / \mathrm{min} / 1.73 \mathrm{~m}^{2}$ (13.6 events per 100 patients-years).

In adjusted models, LV ejection fraction group, LV diameter quartile, pulmonary artery pressure quartile, and RV parameters were independently associated with mortality (Table 3).

\section{Sensitivity analyses}

Adjusted results were qualitatively similar in analyses of the 13,130 outpatients (Additional file 7: Table S7). Results were also similar in those without baseline CHF ( $n=21,036$, Additional file 8: Table S8), except that RV hypertrophy was not significantly associated with mortality, and in patients with available follow-up renal tests or codes $(n=20,008$, Additional file 9: Table S9).

\section{Discussion}

We assessed echocardiographic characteristics of patients with preserved renal function and mild or moderate CKD in a cohort of individuals referred for echocardiography. We found that patients with CKD have a higher prevalence of structural and functional LV and RV abnormalities, which are already apparent in early CKD (eGFR $60-89 \mathrm{ml} / \mathrm{min} / 1.73 \mathrm{~m}^{2}$ ). We also identified that RV systolic function or dilation and pulmonary artery pressure are independently associated with a composite renal outcome of serum creatinine doubling, dialysis, or transplantation. Both RV and LV echocardiographic features were associated with mortality.

Park et al. analyzed the CRIC population and found that decreasing renal function was associated with LV hypertrophy, abnormal LV geometry, diastolic dysfunction, but not with systolic dysfunction [5]. Gori et al. described cardiac structure and function in 217 individuals with heart failure with preserved ejection fraction from the PARAMOUNT trial [3]. They found that renal impairment was associated with abnormal LV geometry, higher LV mass or LV mass index, and lower mid-wall fractional shortening [3]. Creatinine clearance was also identified as an independent predictor of heart failure with preserved ejection fraction in a retrospective study of patients with hypertension and preserved ejection fraction [6]. In a cohort of post-myocardial infarction

Table 3 Adjusted associations of echocardiographic parameters with composite renal outcomes and mortality

\begin{tabular}{|c|c|c|c|c|c|c|c|}
\hline & Parameter & $\begin{array}{l}\text { Number of } \\
\text { renal events }\end{array}$ & $\begin{array}{l}\text { Adjusted HR } \\
\text { (95\% CI) - Renal } \\
\text { outcomes }\end{array}$ & $p$ & Number of deaths & $\begin{array}{l}\text { Adjusted HR } \\
(95 \% \mathrm{Cl})- \\
\text { Mortality }\end{array}$ & $p$ \\
\hline \multirow[t]{3}{*}{ LVEF } & Group 1 vs. 4 & 67 vs. 649 & $1.18(0.88-1.59)$ & 0.26 & 423 vs. 5602 & $1.46(1.31-1.63)$ & $<0.001$ \\
\hline & Group 2 vs. 4 & 91 vs. 649 & $1.06(0.82-1.36)$ & 0.66 & 803 vs. 5602 & $1.27(1.17-1.38)$ & $<0.001$ \\
\hline & Group 3 vs. 4 & 160 vs. 649 & $1.05(0.87-1.26)$ & 0.64 & 1403 vs. 5602 & $1.09(1.02-1.16)$ & 0.007 \\
\hline \multirow[t]{3}{*}{ LVd } & Quartile 2 vs. 1 & 178 vs. 208 & $0.77(0.63-0.95)$ & 0.02 & 1805 vs. 2211 & $0.83(0.78-0.89)$ & $<0.001$ \\
\hline & Quartile 3 vs. 1 & 211 vs. 208 & $0.97(0.79-1.19)$ & 0.78 & 1553 vs. 2211 & $0.80(0.75-0.86)$ & $<0.001$ \\
\hline & Quartile 4 vs. 1 & 292 vs. 208 & $1.02(0.83-1.26)$ & 0.83 & 1872 vs. 2211 & $0.84(0.78-0.90)$ & $<0.001$ \\
\hline \multirow[t]{3}{*}{ LVMi } & Quartile 2 vs. 1 & 42 vs. 56 & $0.72(0.47-1.08)$ & 0.11 & 450 vs. 458 & $0.93(0.81-1.06)$ & 0.29 \\
\hline & Quartile 3 vs. 1 & 84 vs. 56 & $1.29(0.90-1.84)$ & 0.17 & 551 vs. 458 & $0.97(0.85-1.11)$ & 0.65 \\
\hline & Quartile 4 vs. 1 & 63 vs. 56 & $0.81(0.54-1.22)$ & 0.31 & 626 vs. 458 & $0.95(0.83-1.08)$ & 0.41 \\
\hline \multirow[t]{3}{*}{ PAP } & Quartile 2 vs. 1 & 167 vs. 115 & $1.31(1.02-1.67)$ & 0.03 & 1452 vs. 1090 & $1.11(1.02-1.20)$ & 0.02 \\
\hline & Quartile 3 vs. 1 & 157 vs. 115 & $1.67(1.30-2.15)$ & $<0.001$ & 1374 vs. 1090 & $1.26(1.16-1.37)$ & $<0.001$ \\
\hline & Quartile 4 vs. 1 & 223 vs. 115 & $2.26(1.77-2.88)$ & $<0.001$ & 2062 vs. 1090 & $1.73(1.60-1.88)$ & $<0.001$ \\
\hline RV systolic function & Reduced vs. preserved & 106 vs. 743 & $1.51(1.21-1.88)$ & $<0.001$ & 881 vs. 6494 & $1.61(1.49-1.73)$ & $<0.001$ \\
\hline RV hypertrophy & Present vs. absent & 26 vs. 417 & $1.33(0.88-2.02)$ & 0.18 & 204 vs. 3971 & $1.17(1.01-1.35)$ & 0.04 \\
\hline RV dilation & Present vs. absent & 149 vs. 740 & $1.59(1.32-1.92)$ & $<0.001$ & 1189 vs. 6414 & $1.45(1.36-1.55)$ & $<0.001$ \\
\hline
\end{tabular}

Adjusted Cox models. Hazard ratios are adjusted for age, sex, race, baseline eGFR, history of hypertension, diabetes, CAD, or CHF, and use of ACEI and/or ARB. Composite renal outcome includes doubling of serum creatinine or initiation of maintenance dialysis or kidney transplantation. For LVEF, the following cutoffs were used: $<25 \%, 25-39 \%, 40-54 \%, \geq 55 \%$. HR, hazards ratio; Cl, confidence interval; AA, African American race; eGFR, estimated glomerular filtration rate; HTN, hypertension; CAD, coronary artery disease; CHF, congestive heart failure; ACEI, angiotensin converting enzyme inhibitor; ARB, angiotensin receptor blocker; LVEF, left ventricular ejection fraction; LVd, left ventricular diastolic diameter; LVMi, left ventricular mass index (corrected for body surface area); PAP, pulmonary artery pressure; RV, right ventricle 
patients from the VALIANT trial, worse renal function was associated with smaller LV, larger left atrial volume, and higher LV mass index [7]. In contrast, in hypertensive patients with asymptomatic diastolic dysfunction from the VALIDD and EXCEED trials, eGFR group was not associated with echocardiographic measures of LV structure and function [8].

Our findings confirm most of these observations in a much larger population including patients with and without CHF. We also report a significant association of worse renal function with LV systolic impairment, and we expand the previous findings by looking at RV parameters and their association with CKD.

We found that only pulmonary artery pressure and parameters related to the RV are associated with CKD progression. The AASK study addressed this issue in African-American patients with pre-existing non-diabetic hypertensive CKD and showed that LV mass index was not associated with a combined outcome of doubling of creatinine or end-stage renal disease [9]. On the contrary, a retrospective analysis of the Jackson Heart Study, also in an African-American population, showed a significant association of LV mass, but not LV ejection fraction or pulmonary artery pressure, with a composite outcome of eGFR decline $>30 \%$ or progression to end-stage renal disease [10]. A small cohort study in stage 3-5 CKD patients demonstrated that concentric LV hypertrophy was associated with progression to dialysis, whereas LV ejection fraction and increased left atrium diameter were associated with CKD progression, defined as eGFR decline $>3 \mathrm{ml} /$ $\mathrm{min} / 1.73 \mathrm{~m}^{2}$ annually $[11,12]$. In addition, a study in 300 elderly patients showed that LV hypertrophy was independently associated with eGFR decline $>5 \mathrm{ml} / \mathrm{min} /$ $1.73 \mathrm{~m}^{2}$ per year [13]. In the ESCAPE trial, a significant correlation was identified between baseline creatinine levels and right atrial pressure levels in patients admitted with heart failure [14]. Park et al., using magnetic resonance imaging, demonstrated that LV mass and concentricity, a measurement of abnormal cardiac size, were associated with eGFR decline and incident CKD [15].

Discrepancies with our findings may reflect differences in baseline renal and heart function, demographic characteristics and the use of a clinical cohort in our study compared to highly selected prospective research or trial cohorts in the prior studies. Furthermore, renal outcomes were not defined in the same way. Lack of association between LV mass index and renal outcomes or mortality in our study may be due to values below hypertrophic levels for the majority of individuals. LV mass could be more reliably assessed with more advanced techniques, such as magnetic resonance imaging. Similarly, lack of association between pulmonary artery pressure and renal outcomes in the Jackson Heart Study may be due to relatively normal pulmonary artery pressure values among study participants.
Our findings extend these findings by demonstrating a significant, independent association between RV diameter or function and CKD progression in a population that primarily did not have CHF. Worsening RV function and elevated pulmonary artery pressures may be associated with higher renal vein pressures, a purported mechanism underlying the cardiorenal syndrome [16, 17]. Similarly, salt and water retention in the setting of decreasing eGFR may contribute to volume overload and increased renal vein pressures that may impair renal perfusion and cause ischemia-mediated sclerotic changes. On the contrary, LV dysfunction may not significantly impact renal function provided that renal perfusion pressure remains in an acceptable range. However, these findings are only hypothesis generating and need to be prospectively confirmed. Finally, we also report a significant association between mortality and abnormal RV structure or function. No prior data exist on the association between right heart parameters and mortality in patients with CKD, to our knowledge.

Our study has several limitations. It is a retrospective study of patients referred for echocardiography and may not reflect the complete spectrum of patients with preserved renal function or mild eGFR impairment since sicker patients are more likely to be referred for echocardiography and those with more advanced cardiopulmonary disease are more likely to have RV dysfunction. Furthermore, no information was available on the indication for the echocardiogram. Echocardiograms were ordered to answer specific clinical questions rather than to provide systemic assessment of cardiac structure and they were interpreted by multiple clinicians rather than by a core lab, raising the possibility of confounding introduced by wide inter-reader variability between sonographers. In particular, RV assessment is fraught with potential errors of judgment and high degree of inter-observer variability. Additionally, tissue Doppler imaging results, diastolic function, and valve structure and function were not extractable for most patients.

Index echocardiograms might have been conducted in the setting of decompensated CHF and may not reflect baseline cardiac status or could have been followed by acute changes in kidney function. However, our findings were qualitatively similar in a subgroup analysis including only outpatient echocardiograms in which these issues would be less likely. We similarly cannot rule out the possibility that addition of renin-angiotensin-system agents in individuals with more severe echocardiographic changes partly accounted for associations with CKD progression, although our results were adjusted for use of these agents at baseline. Furthermore, the clinical indication of the echocardiogram may have already caused changes in eGFR. Capture of medication changes during follow-up is not readily available to us at this time. Finally, there were no BP or proteinuria values available for most patients in this dataset, and no information was available on the cause 
of death. Nevertheless, a sample size several times larger than all prior cohorts, assessment of multiple echocardiographic parameters, and most importantly a sufficient number of outcomes to be powered to examine associations with CKD progression and mortality represent unique strengths of our analysis.

More studies are needed to prospectively confirm the identified associations and carefully evaluate the right heart with comprehensive assessment of the RV including lateral tricuspid annular systolic velocity and acceleration, fractional area and long-axis change, tricuspid annular systolic excursion, myocardial performance index, and lateral free wall peak systolic strain, as well as inferior vena cava diameter and distensibility $[18,19]$. These results also merit analysis in the setting of advanced CKD $\left(e G F R<30 \mathrm{ml} / \mathrm{min} / 1.73 \mathrm{~m}^{2}\right)$.

\section{Conclusions}

In conclusion, structural and functional abnormalities of the right and left ventricle are more common in individuals with mild or moderate renal impairment and associated with mortality while RV function and structure are independently associated with CKD progression even in early stage CKD. Prospective studies with quantitative assessment of the RV are needed to validate our observations and better understand the underlying mechanisms.

\section{Additional files}

Additional file 1: Table S1. Condition specific diagnostic codes (International Classification of Diseases, 9th revision) (DOCX $92 \mathrm{~kb}$ ).

Additional file 2: Table S2. Crude and adjusted assessment of echocardiographic parameters and estimated glomerular filtration rate (DOCX $17 \mathrm{~kb}$ ).

Additional file 3: Table S3. Baseline characteristics of patients with $\mathrm{CHF}$ at baseline (DOCX $18 \mathrm{~kb}$ ).

Additional file 4: Table S4. Echocardiographic characteristics of patients with CHF at baseline (DOCX $17 \mathrm{~kb})$.

Additional file 5: Table S5. Baseline characteristics of patients without CHF at baseline (DOCX $17 \mathrm{~kb}$ ).

Additional file 6: Table S6. Echocardiographic characteristics of patients without CHF at baseline (DOCX $16 \mathrm{~kb}$ ).

Additional file 7: Table S7. Adjusted associations of echocardiographic parameters with composite renal outcomes and mortality in outpatients (DOCX $16 \mathrm{~kb})$.

Additional file 8: Table S8. Adjusted associations of echocardiographic parameters with composite renal outcomes and mortality in patients without known CHF at baseline (DOCX $16 \mathrm{~kb}$ )

Additional file 9: Table S9. Adjusted associations of echocardiographic parameters with composite renal outcomes and mortality in patients with available follow-up creatinine values or diagnostic codes (DOCX $16 \mathrm{~kb}$ ).

\section{Abbreviations}

ACEl: angiotensin converting enzyme inhibitors; ARB: angiotensin receptor blockers; BNP: brain natriuretic peptide; CHF: congestive heart failure; CKD: chronic kidney disease; eGFR: estimated glomerular filtration rate; FDA: Food and Drug Administration; LV: left ventricular; RPDR: Research Patient Data registry; RV: right ventricular
Funding

Drs. Khattak and Singh were supported by the NIDDK training grant T32DK007527

\section{Availability of data and materials}

The data that support the findings of this study are available from Partners RPDR but restrictions apply to the availability of these data, which were used under license for the current study, and so are not publicly available. Data are however available from the authors upon reasonable request and with permission of Partners IRB.

\section{Authors' contributions}

AK \& DC designed the study. AK \& KS collected the data. TM \& DC analyzed and interpreted the data. TM drafted the first version of the manuscript. AK, $\mathrm{KS}$, and DC critically reviewed the manuscript. DC supervised the study. All authors read and approved the final version of the manuscript.

Ethics approval and consent to participate

The Partners Healthcare institutional review board approved the study and waived the need for consent.

\section{Consent for publication}

Not applicable.

\section{Competing interests}

The authors declare that they have no competing interests.

\section{Publisher's Note}

Springer Nature remains neutral with regard to jurisdictional claims in published maps and institutional affiliations.

\section{Author details}

${ }^{1}$ Renal Division, Brigham \& Women's Hospital, Harvard Medical School, Boston, MA, USA. 'Division of Nephrology, Department of Medicine, McGill University, Montreal, Canada. ${ }^{3}$ Division of Renal Disease and Hypertension, Department of Internal Medicine, The University of Texas Health Science Center, Houston, TX, USA. ${ }^{4}$ Departments of Learning Health Sciences and Internal Medicine, University of Michigan Medical School, Ann Arbor, MI, USA.

Received: 10 April 2018 Accepted: 27 June 2018

Published online: 11 July 2018

\section{References}

1. McAlister FA, Ezekowitz J, Tonelli M, Armstrong PW. Renal insufficiency and heart failure: prognostic and therapeutic implications from a prospective cohort study. Circulation. 2004;109:1004-9.

2. Hillege HL, Nitsch D, Pfeffer MA, Swedberg K, McMurray JJ, Yusuf S, et al. Renal function as a predictor of outcome in a broad spectrum of patients with heart failure. Circulation. 2006;113:671-8.

3. Gori M, Senni M, Gupta DK, Charytan DM, Kraigher-Krainer E, Pieske B, et al. Association between renal function and cardiovascular structure and function in heart failure with preserved ejection fraction. Eur Heart J. 2014;35:3442-51.

4. Untersteller K, Girerd N, Duarte K, Rogacev KS, Seiler-Mussler S, Fliser D, et al. NT-proBNP and echocardiographic parameters for prediction of cardiovascular outcomes in patients with CKD stages G2-G4. Clin J Am Soc Nephrol. 2016;11:1978-88.

5. Park M, Hsu CY, Li Y, Mishra RK, Keane M, Rosas SE, et al. Associations between kidney function and subclinical cardiac abnormalities in CKD. J Am Soc Nephrol. 2012;23:1725-34.

6. Nishio M, Sakata Y, Mano T, Ohtani T, Takeda Y, Hori M, et al. Difference of clinical characteristics between hypertensive patients with and without diastolic heart failure: the roles of diastolic dysfunction and renal insufficiency. Hypertens Res. 2008;31:1865-72.

7. Verma A, Anavekar NS, Meris A, Thune JJ, Arnold JM, Ghali JK, et al. The relationship between renal function and cardiac structure, function, and prognosis after myocardial infarction: the VALIANT Echo Study. J Am Coll Cardiol. 2007:50:1238-45.

8. Shah AM, Lam CS, Cheng S, Verma A, Desai AS, Rocha RA, et al. The relationship between renal impairment and left ventricular structure, function, and ventricular-arterial interaction in hypertension. J Hypertens. 2011;29:1829-36. 
9. Peterson GE, de Backer T, Contreras G, Wang X, Kendrick C, Greene T, et al. Relationship of left ventricular hypertrophy and diastolic function with cardiovascular and renal outcomes in African Americans with hypertensive chronic kidney disease. Hypertension. 2013:62:518-25.

10. Zelnick LR, Katz R, Young BA, Correa A, Kestenbaum BR, de Boer $\mathbb{H}$, et al. Echocardiographic measures and estimated GFR decline among African Americans: the Jackson heart study. Am J Kidney Dis. 2017;70:199-206.

11. Chen SC, Su HM, Hung CC, Chang JM, Liu WC, Tsai JC, et al. Echocardiographic parameters are independently associated with rate of renal function decline and progression to dialysis in patients with chronic kidney disease. Clin J Am Soc Nephrol. 2011;6:2750-8.

12. Chang JM, Chen SC, Huang JC, Su HM, Chen HC. Anemia and left ventricular hypertrophy with renal function decline and cardiovascular events in chronic kidney disease. Am J Med Sci. 2014;347:183-9.

13. Shi HT, Wang XJ, Li J, Song GF, Huang ZY, Guo XY, et al. Association of Left Ventricular Hypertrophy with a faster rate of renal function decline in elderly patients with non-end-stage renal disease. J Am Heart Assoc. 2015;4:e002213.

14. Nohria A, Hasselblad V, Stebbins A, Pauly DF, Fonarow GC, Shah M, et al. Cardiorenal interactions: insights from the ESCAPE trial. J Am Coll Cardiol. 2008;51:1268-74

15. Park M, Shlipak MG, Katz R, Agarwal S, IX JH, Hsu C, et al. Subclinical cardiac abnormalities and kidney function decline: the multi-ethnic study of atherosclerosis. Clin J Am Soc Nephrol. 2012;7:1137-44.

16. Mullens W, Abrahams Z, Francis GS, Sokos G, Taylor DO, Starling RC, et al. Importance of venous congestion for worsening of renal function in advanced decompensated heart failure. J Am Coll Cardiol. 2009:53:589-96.

17. Braam B, Cupples WA, Joles JA, Gaillard C. Systemic arterial and venous determinants of renal hemodynamics in congestive heart failure. Heart Fail Rev. 2012;17:161-75.

18. Pavlicek M, Wahl A, Rutz T, de Marchi SF, Hille R, Wustmann K, et al. Right ventricular systolic function assessment: rank of echocardiographic methods vs. cardiac magnetic resonance imaging. Eur J Echocardiogr. 2011;12:871-80.

19. Rudski LG, Lai WW, Afilalo J, Hua L, Handschumacher MD, Chandrasekaran K, et al. Guidelines for the echocardiographic assessment of the right heart in adults: a report from the American Society of Echocardiography endorsed by the European Association of Echocardiography, a registered branch of the European Society of Cardiology, and the Canadian Society of Echocardiography. J Am Soc Echocardiogr. 2010;23:685-713.

\section{Ready to submit your research? Choose BMC and benefit from:}

- fast, convenient online submission

- thorough peer review by experienced researchers in your field

- rapid publication on acceptance

- support for research data, including large and complex data types

- gold Open Access which fosters wider collaboration and increased citations - maximum visibility for your research: over $100 \mathrm{M}$ website views per year

At BMC, research is always in progress.

Learn more biomedcentral.com/submissions 